\title{
An ID-based authenticated three-party key exchange protocol
}

\author{
Susmita Mandal", Sujata Mohanty and Banshidhar Majhi \\ Department of Computer Science and Engineering, National Institute of Technology, Rourkela, Odisha
}

\section{(C)2017 ACCENTS}

\begin{abstract}
For secure communication in an open and distributed network, three-party authenticated key exchange (3PAKE) protocol establishes a secure session key between two users with the help of a trusted server to ensure transaction confidentiality and efficiency. Existing schemes fail to achieve privacy to user's identity also unable to ensure undeniability of a service request. Therefore, we propose an authenticated three-party key exchange scheme based on the elliptic curve computational diffie-hellman assumption (ECDH). The proposed scheme not only achieves anonymity, nonrepudiation but also reduces the overall computational cost. The scheme is validated in automated validation of internet security protocols and applications (AVISPA) tool and is proved secure in the random oracle model. The scheme has huge applications in real life scenarios, namely, mobile-commerce; secure message transmission, and e-voting.
\end{abstract}

Keywords

Anonymity, Non-repudiation, ID-based, Elliptic curve cryptography, AVISPA tool.

\section{Introduction}

Key exchange protocols enable two parties to communicate securely over an untrusted network by exchanging a shared secret among them. Authentication and privacy are the two primary objectives of network security where privacy ensures that transmitted messages cannot eavesdrop. On the other hand, authentication assures that no unauthorized user can gain access maliciously. These two goals can be achieved simultaneously, using authenticated key exchange scheme where two or more parties can share a common secret to transmit a message securely in an open network.

Bellovin and Merritt [1] proposed a primitive twoparty password-based authentication key exchange (2PAKE) protocol where each entity can authenticate one another via a public network for sharing a session key. On the basis of their protocol, several 2PAKE protocols [2-4] are proposed in the literature. However, the 2PAKE protocols are mostly suitable for client-server architectures, as they need to preshare a common secret for mutual authentication and session key agreement. This restriction results in storage of huge amount of secret for communicating with a group of participants.

\footnotetext{
*Author for correspondence
}

To overcome this problem, $3 P A K E$ schemes are proposed where every user shares a single secret with a trusted server by eliminating the necessity of holding a huge amount of secrets to communicating with different group members. The first efficient three-party authenticated key exchange protocol based on $P K C$ was proposed by Chen et al. [5]. The scheme proposes low round complexity to achieve mutual authentication. Yang and Chang [6] found, Chen's protocol suffer from the stolen-verifier attack and require more computation cost as it generates and verifies Schnorr's [7] digital signature based on modular exponentiation. Then, Yang et al. proposed an improved $3 P A K E$ protocol based on elliptic curve cryptography $(E C C)$ without any pre-shared secrets. Later, Yang et al. proposed an improved $3 P A K E$ protocol based on elliptic curve cryptography $(E C C)$ without any pre-shared secrets between client and server resulting in lower computation costs and low communication loads. In 2009,2010, Pu et al. and Tan found that Yang's scheme is vulnerable to unknown key-share attack, man-in-the-middle attack, impersonation, and parallel session attack [8, 9]. In the same paper, Tan et al. proposed a modified $3 P A K E$ using the ECC but recently, Nose [10] proved that Tan's protocol suffers from impersonation and the man-in-the-middle attack.

Abundant work has been done in $3 P A K E$ relevant to password-based authentication, traditional public key cryptosystem $(P K C)$ and without server's public key 
[11-14]. This causes very high computation loads and the communication costs of transmission. To simplify the complexity of certificate management Shamir [15] introduced ID-based cryptosystem where the public keys of each user are easily computed using user's identity. To solve the issue of high computational and communication load, elliptic curve cryptography $(E C C)$ is a suitable solution. $E C C$ was first proposed by Miller [16] and Koblitz [17] and its security was based upon the difficulty of solving elliptic curve discrete logarithm problem $(E C D L P)$. Compared with traditional public key cryptosystem, $E C C$ provides better performance as it can achieve the same security with a smaller key size.

\subsection{Our contribution}

In literature, several $3 P A K E$ protocols were proposed based on $P K C$ and modular exponentiation which suffers from public key management and high computational cost. Therefore, we propose an IDbased authenticated three-party key exchange protocol based on ECC in this paper. The characteristics of our, scheme is described as follows: 1. Avoidance of certificate management: Since traditional public key cryptosystem used to verify and manage the certificates before establishing a connection between a client and server. However, it suffers from high computation cost; therefore, the ID-based cryptosystem is an ideal solution to eliminate the certificates.

2. Security analysis: The proposed $3 P A K E$ protocol employs simple hash function, signature analysis, and a single symmetric encryption/decryption process. The security is based on the hardness assumption of elliptic curve computational diffiehellman $(E C D H)$ also it is validated using random oracle model AVISPA.

3. Anonymity and Non-repudiation: It is very important to preserve user privacy in the applications like mobile-commerce, e-voting, and secure message transmission, etc., so that an adversary cannot track user's activity. Therefore, anonymity preserves user's personal information. Similarly, non-repudiation is one of the important requirements for a security protocol in any communication network to prevent denial of conducting an operation on a credit card purchase or any on-line transaction.

\subsection{Organization of the paper}

The rest of the paper is organized as follows. A brief review of some basic concepts is described in Section 2. In section 3, we propose our ID-based three-party authenticated key exchange protocol. The security and analysis of the proposed protocol are presented in Section 4. In section 5 performance analysis is depicted. Finally, we conclude in Section 6.

\section{Preliminaries}

This section introduces two cryptographic techniques used in our proposed scheme: Elliptic curve cryptography and Computational problem.

\subsection{Elliptic curve cryptography}

The security of ECC is based upon the difficulty of (ECDLP).

Let $\mathrm{E} / \mathrm{F}_{q}$ be a set of elliptic curve points over a finite field $\mathrm{F}_{q}$, defined by an equation $y^{2}=x^{3}+a x+b$, $a, b \in F_{p}$ where $\left(4 a^{3}+27 b^{2}\right) \neq 0$. The additive elliptic curve group defined as $G_{q}=\left\{(x, y): x, y \in F_{q},(x, y) \in E / F_{q}\right\} \cup\{O\}$, where the point " $O$ " is known as "point at infinity" or "zero point". The definitions about the elliptic curve group as follows.

1 Point Addition: Let $P, Q$ be two points on the curve shown above, such that $P+Q=R$, where the line joining $P$ and $Q$ intersects the curve at negative $R$, and the reflection towards $x$-axis is $R$.

2 Scalar Point Multiplication: It is defined on a cyclic group $G_{q} \quad$ as $(r P=P+P+\ldots)(+P(r$ times $))$, where $k \in Z_{q}^{*}$ is scalar.

\subsection{Computational problem}

Definition1. (Elliptic curve discrete logarithm problem (ECDLP)) Given $P, R \in G_{q}$, where $R=x P$ and $x \in Z_{q}^{*}$. It is difficult to compute $x$ from $R$.

Definition2. (Computational Diffie-Hellman problem $(\mathrm{ECDH}))$ Given $(P, x P, y P) \in\left(G_{q}\right)$ for $x, y \in Z_{q}^{*}$, where computation of $x y P$ is hard from the group $G_{q}$

\section{Proposed 3PAKE Protocol}

In this section, we propose our ID-based 3PAKE protocol based on ECC. The scheme is divided into three phases: the system initialization phase, the user registration phase, the authenticated key exchange phase.

3.1Initialization phase

In this phase, the server $\mathrm{S}$ generates set of system parameters as follows: 
Susmita Mandal et al.

1. For this, $\mathrm{S}$ chooses an elliptic curve equation $\left(E_{p}(a, b) \in F_{q}\right)$ as defined in Subsection 2.1.

2. The base point $\mathrm{P}$ over $\left(E_{p}(a, b)\right)$ with the order $n$ and chooses a private key $\left(d_{s} \in Z_{n}^{*}\right)$ and the corresponding public key $\left(Q_{s}=d_{s}\right)$.

3 . The server chooses three secure one-way hash functions $\left(H_{1}(),. H_{2}(),. H_{3}().\right) .\left(G_{p}\right)$ represents a cyclic addition group that is generated by $(P)$ over $\left(E_{p}(a, b)\right)$.

4. The server keeps $\left(d_{s}\right)$ secret and publishes $\left(\left\{E_{p}(a, b), P, Q_{s}, H_{1}(),. H_{2}(),. H_{3}().\right\}\right)$

\subsection{Registration}

In this phase, any new user participating in the communication need to register with the server S. The user sends its identity in advance. Later, the server computes a temporary identity known only to designated user and server for preserving anonymity. The user and server perform following steps via a secure channel as follows.

1. The server chooses an integer randomly $\left(r_{u} \in Z_{p}^{*}\right)$. and computes, $\left(R_{u}=r_{u} P\right)$.

2. S computes $\left(h_{u}=H_{1}\left(I D_{u}\left\|I D_{s}\right\| R_{u}\right)\right)$, then computes client's private key $\left(U=r_{u}+h_{u} \cdot d_{s}\right)$.

3. Thereafter, computes a temporary identity $\left(T I D_{u}=H_{2}\left(d_{s}\right) \oplus H_{2}\left(I D_{u}\right)\right)$ and generates a random nonce $\mathrm{N}_{\mathrm{s}}$.

4. Later, $\mathrm{S}$ sends $\left(\left\{I D_{s}, U, R_{u}, T I D_{u}, N_{s}\right\}\right)$ to every client.

5. Upon receiving from server the client verifies $\left(U . P \stackrel{?}{=} R_{u}+H_{1}\left(I D_{u}\left\|I D_{s}\right\| R_{u}\right) \cdot Q_{s}\right)$ if it holds, the client keeps $\mathrm{U}$ and publishes $\left(R_{u}\right)$.

\subsection{Authentication phase}

In this phase, two entities $\mathrm{A}$ and $\mathrm{B}$ wants to authenticate each other via a server $\mathrm{S}$ and generate a session key for future communication, assuming that $\mathrm{A}$ as a sender and $\mathrm{B}$ as a receiver. The steps are presented as follows.

Round 1.

1. In this round the initiator $A$ randomly picks $\left(x_{a} \in Z_{p}^{*}\right)$ as its ephemeral-private-key, then computes $\left(P_{u a}=x_{a} \cdot P\right)$. Thereafter, he computes $\left(t_{1}=H_{2}\left(T_{1}\right)\right)$ where $\mathrm{T}_{1}$ is a time-stamp which denotes the current time.

2. To preserve the anonymity the user A computes following parameters to hide its identity as, $\left(V_{a}=T I D_{a} \oplus H_{2}\left(I D_{a}\right)\right), \quad\left(M_{a}=V_{a} \oplus U_{a}\right)$, $\left(Z_{a}=H_{1}\left(V_{a} \| U_{a}\right) \oplus I D_{a}\right)$. Further, he computes $\left(F_{a}=V_{a} \oplus X_{a}\right)$ where, $\left(X_{a}=H_{1}\left(x_{a} \| I D_{a}\right)\right)$.

3. Then, a shared session key is computed as $\left(S K_{a s}=H_{3}\left(T I D_{a}\left\|I D_{s}\right\| R_{a}\left\|X_{a}\right\| V_{a}\right)\right)$ followed by a symmetric key encryption is generated as $\left(K_{a}=\left(K_{a}=H_{3}\left(T I D_{a}\left\|T I D_{b}\right\| I D_{s}\left\|R_{a}\right\| t_{1}\right)\right)\right.$.

4. Then user A sends $\left(\left(T I D_{a}, N_{a}\right.\right.$, Request $\left.)\right)$, $\left(\left(T I D_{a}, T I D_{b}, I D_{s}, T_{1}\right.\right.$,

$\left.\left.E_{K_{a}}\left[V_{a}, M_{a},\right)\left(F_{a}, S K_{a s}\right]\right)\right)$ to $\mathrm{B}$ and $\mathrm{S}$ respectively.

5. Similarly, B computes and send $\left(\left(T I D_{b}, N_{b}\right.\right.$, Response $\left.)\right)$,

$\left(\left(T I D_{b}, T I D_{a}, I D_{s}, T_{2},\right)\left(E_{K_{b}}\right)\right)$ to $\mathrm{A}$ and $\mathrm{S}$ respectively.

Round 2.

1. Upon receiving A's initiation message the server $S$ computes $\left(t_{1}=H_{2}\left(T_{1}\right)\right)$.

2. Then apply a decryption algorithm on $\left(D_{K_{a}}\right)$ as $\left(K_{a} \stackrel{?}{=} H_{3}\left(T I D_{a}\left\|T I D_{b}\right\| I D_{s}\right)\left(\left\|R_{a}\right\| t_{1}\right)\right)$. If $\quad$ it holds then check if $\left(V_{a} \stackrel{?}{=} H_{2}\left(d_{s}\right)\right)$, if verified then compute $\left(X_{a}=F_{a} \oplus H_{2}\left(d_{s}\right)\right)$.

3. Upon confirmation $\mathrm{S}$ compute the shared common session key as $\left(S K_{a s}=H_{3}\left(T I D_{a}\left\|I D_{s}\right\| R_{a}\left\|X_{a}\right\| H_{2}\left(d_{s}\right)\right)\right)$.

4. After successful verification $S$ computes $\left(C_{s a}=H_{3}\left(T I D_{a}\left\|T I D_{b}\right\| R_{a}\left\|R_{b}\right\| P_{u a}\right)\left(\left\|P_{u b}\right\| S K_{s a}\right)\right)$ for user A. Similarly, compute $\left(C_{s b}=H_{3}\left(T I D_{a}\left\|T I D_{b}\right\| R_{a}\left\|R_{b}\right\|\right)\left(P_{u a}\left\|P_{u b}\right\| S K_{s b}\right)\right)$ for user B.

5. After which, S sends $\left(\left(I D_{s}, T I D_{b}, P_{u b}, E_{K_{s a}}\left[R_{b}, C_{s a}\right]\right)\right)$ to user A where the $\quad \operatorname{key}\left(K_{s a}=H_{3}\left(I D_{s}\left\|T I D_{b}\right\| H_{2}\left(d_{s}\right) \| P_{u b}\right)\right)$.

Similarly, sends 
$\left(\left(I D_{s}, T I D_{a}, P_{u a},\right)\left(E_{K_{s b}}\left[R_{a}, C_{s b}\right]\right)\right)$ to user $\mathrm{B}$ where $\left(K_{s b}=H_{3}\left(I D_{s}\left\|T I D_{a}\right\| H_{1}\left(d_{s}\right) \| P_{\text {иа }}\right)\right)$.

Round 3.

1. Upon receiving, user A first applies a decryption algorithm

$\left(K_{s a} \stackrel{?}{=} H_{3}\left(I D_{s} \|\right)\left(T I D_{b}\left\|H_{2}\left(d_{s}\right)\right\| P_{u b}\right)\right)$.

2. Then

verifies

$\left(C_{s a} \stackrel{?}{=} H_{3}\left(T I D_{a}\left\|T I D_{b}\right\| R_{a}\left\|R_{b}\right\| P_{u a}\left\|P_{u b}\right\| S K_{a s}\right)\right)$ if it holds then user A generate a time-stamp $\mathrm{T}_{3}$ then compute $\left(t_{3}=H_{2}\left(T_{3} \| N_{b}\right)\right)$ where $\mathrm{N}_{\mathrm{b}}$ is a random nonce sent by $\mathrm{B}$ in Round 1 .

3. A signature is generated to prevent repudiation by a legitimate but curious user as $\left(\sigma_{a}=\left(x_{a}+t_{3}\right) \cdot P_{u b}\right)$ followed by a common shared $\operatorname{key}\left(K_{a b}=x_{a} \cdot P_{u b}\right)$.

4. Thereafter, A send $\left(\left(T I D_{a}, T I D_{b}, E_{K_{a b}}\left[T_{3}, \sigma_{a}, N_{b}\right]\right)\right)$ to $\mathrm{B}$.

5. Later, compute the shared session key $\left(K=H_{1}\left(K_{a b}\left\|N_{a}\right\| N_{b}\left\|R_{a}\right\| R_{b} \| \sigma_{a}\right)\right)$ and the session

$\left(S K_{a b}=H_{3}\left(T I D_{a}\left\|T I D_{b}\right\| P_{u a}\left\|P_{u b}\right\| R_{a}\left\|R_{b}\right\| K\right)\right)$ key

further communication.

Round 4.

1. After receiving B computes $\left(K_{a b}=x_{b} \cdot P_{u a}\right)$ then applies a decryption algorithm.

2. Then computes $\left(t_{3}=H_{2}\left(T_{3} \| N_{b}\right)\right)$ and verifies the signature as $\left(\sigma_{a}=\left(P_{u a}+t_{3} \cdot P\right) \cdot x_{b}\right)$, if it holds then compute a session key $\left(S K_{a s}=H_{3}\left(T I D_{a}\left\|T I D_{b}\right\| P_{u a}\right)\left(\left\|P_{u b}\right\| R_{a}\left\|R_{b}\right\| K\right)\right)$ where $\left(K=H_{1}\left(K_{a b}\left\|N_{a}\right\| N_{b}\left\|R_{a}\right\| R_{b} \| \sigma_{a}\right)\right)$ for $\quad$ future communication.

\section{Security model}

\subsection{Security analysis}

This section provides the provable security analysis in random oracle model [18] against chosen message attack and a formal security verification of the proposed 3PAKE scheme using AVISPA tool.
Theorem 1. The user with identity $\left(I D_{u} \in\{A, B\}\right)$ accepts the private key $\mathrm{U}$ if $\left(U . P=R_{u}+H_{1}\left(I D_{U}\left\|I D_{S}\right\| R_{u}\right) \cdot Q_{s}\right)$ holds.

Proof. The correctness of the verification phase is proved as follows:

The user receive $\left(R_{u}, U\right)$ along with server's ID $\left(I D_{s}\right)$ then he computes

$$
\begin{aligned}
& \left.U . \mathrm{P}=R_{u}+H_{1}\left(I D_{u}\left\|I D_{s}\right\| R_{u}\right) \cdot Q_{s}\right) \\
& \left.=r_{u} \cdot P+h_{A} \cdot d_{s} \cdot P\right) \\
& =U \cdot P
\end{aligned}
$$

Theorem 2.The signer $\mathrm{ID}_{\mathrm{a}}$ generates the signature $\left(\sigma_{a}\right)$ then send to $\mathrm{B}$ who can accept if his authenticity is proved based on the condition if $\left(\sigma_{a}=\hat{\sigma}_{a}\right)$ holds.

Proof. The correctness of the verification phase is proved as follows:

$\mathrm{B}$ receives $\left(\left[T_{3}, \sigma_{a}, N_{a}\right]\right)$ using which he computes following parameters $\left(\hat{t}_{3}\right)$ then computes

$$
\begin{aligned}
& \left.\hat{\sigma}_{a}=\left(P_{u a}+\hat{t}_{3} \cdot P\right) \cdot x_{b}\right) \\
& \left.=\left(x_{a} \cdot x_{b} \cdot P+\hat{t}_{3} \cdot P \cdot x_{b}\right)\right) \\
& \left.=\left(x_{a} \cdot P_{u b}+\hat{t}_{3} \cdot P_{u b}\right)\right) \\
& \left.=\left(x_{a}+\hat{t}_{3}\right) \cdot P_{u b}\right)
\end{aligned}
$$

Theorem 3 The identity of a participated user should not be revealed even if an adversary tries to read a transmitted message and also it should not be distinguishable if either two different sessions are initiated by same user then, the scheme achieves anonymity.

Proof. In our protocol, the identity of user $(\in\{A, B\})$ is hidden $\operatorname{in}\left(Z_{a}=H_{1}\left(V_{a} \| U_{a}\right) \oplus I D_{a}\right)$, which contains the private key generated by $\mathrm{S}$ which is initiated at each session and is protected by secret $\operatorname{key}\left(V_{a}=H_{2}\left(d_{s}\right)\right)$. Therefore, the message is fresh in each session and even if an adversary captures the identity $T I D_{u}$ he will be unable to link any two sessions executed before. 
Susmita Mandal et al.

Theorem 4 The proposed 3PAKE protocol is provably secure against adaptively chosen message attack in the random oracle under the Elliptic curve computational diffie-hellman (ECDH) assumption. Proof. Assume that the proposed 3PAKE scheme can be forged under the adaptive chosen message attack by a probabilistic polynomial time adversary whose goal is to intrude into the system and break our scheme. For the same, we construct an algorithm $\mathrm{C}$ that helps an adversary A to solve the $(\mathrm{ECDH})$ problem by responding with $(x y P)$ as output from $(P, x P, y P)$, where $x, y \in Z_{p}^{*}$.

Initialization: Initially, $\mathrm{C}$ chooses a $Q_{c} \in Z_{p}^{*}$ to solve the $(\mathrm{ECDH})$ problem, by setting $Q_{s}=Q_{c}$ and finally $\mathrm{C}$ gives returns the system parameters $\backslash\left(\left\{E_{p}(a, b), P, Q_{c}, H_{1}(),. H_{2}(),. H_{3}().\right\}\right)$ to $\mathrm{A}$ and responses the queries made by $\backslash(\mathrm{A} \backslash)$ as follows: $\backslash \backslash$

Hash queries to $\left(\mathbf{H}_{1}\right)$ : Initially, $\mathrm{C}$ holds an empty $\left(L_{H_{1}}^{\text {list }}\right)$ list. Each entry in the list is a tuple of the form $\left(\left(I D_{u}, I D_{s}, R_{u}, h_{u}\right)\right), \quad\left(\left(V_{u}, U_{u}, Z_{u}\right)\right)$, $\left(\left(x_{u}, I D_{u}, X_{u}\right)\right),\left(K_{i j}, N_{i}, N_{j}, R_{i}, R_{j}, \sigma_{i}, K_{i}\right)$.For each query $\left(\left(I D_{u}, I D_{s}, R_{u}, V_{u}, U_{u}, x_{u}, I D_{u}\right)\right)$ issued by adversary A to the oracle, $\mathrm{C}$ either returns a previous value else choose a number $\left(h_{u}, Z_{u}, X_{u}, K_{i} \in Z_{p}^{*}\right)$ such that there is no item $\left(\left(., ., ., h_{u}\right)\right),\left(\left(., ., Z_{u}\right)\right),\left(\left(., ., X_{u}\right)\right),\left(\left(., ., ., ., . ., ., K_{i}\right)\right) \operatorname{in}\left(L_{H_{1}}^{\text {list }}\right)$ and returns $\left(h_{u}, Z_{u}, X_{u}, K_{i}\right)$ to $\mathrm{A}$. Now $\mathrm{C}$ inserts the tuple $\left(\left(I D_{u}, I D_{s}, R_{u}, h_{u}\right)\right), \quad\left(\left(V_{u}, U_{u}, Z_{u}\right)\right)$, $\left(\left(x_{u}, I D_{u}, X_{u}\right)\right), \quad\left(K_{i j}, N_{i}, N_{j}, R_{i}, R_{j}, \sigma_{i}, K_{i}\right)$ in the $\left(L_{H_{1}}^{\text {list }}\right)$.

Hash queries to $\left(\mathbf{H}_{2}\right)$ : Initially, $\mathrm{C}$ holds an empty $\left(L_{\mathrm{H}_{2}}^{\text {list }}\right)$ list. Each entry in the list is a tuple of the form $\left(\left(d_{s}, I D_{u}, T I D_{u}\right)\right),\left(\left(T_{i}, N_{i}, t_{i}\right)\right),\left(\left(I D_{u}, V_{u}\right)\right)$. For each query $\$\left(T I D \_u, t \_i, V \_u\right) \$$ issued by adversary A to the oracle, $\mathrm{C}$ either returns a previous value else choose a number $\left(T I D_{u}, t_{i}, V_{u} \in Z_{p}^{*}\right)$ such that there is no item $\left(\left(., ., T I D_{u}\right)\right),\left(\left(., ., t_{i}\right)\right),\left(\left(., V_{u}\right)\right) i n L_{H_{2}}^{\text {list }}$ and returns $\left(T I D_{u}, t_{i}, V_{u}\right)$ to $\mathrm{A}$. Now $\mathrm{C}$ inserts the 66 tuple $\left(\left(d_{s}, I D_{u}, T I D_{u}\right)\right),\left(\left(T_{i}, N_{i}, t_{i}\right)\right),\left(\left(I D_{u}, V_{u}\right)\right)$ in the $L_{\mathrm{H}_{2}}^{\text {list }}$.

Hash queries to $\mathbf{H}_{3}$ : Initially, $\mathrm{C}$ holds an empty $L_{H_{3}}^{\text {list }}$ list. Each entry in the list is a tuple of the form $\left(\left(T I D_{u}, I D_{s}, R_{u}, X_{u}, V_{u}, S K_{u}\right)\right)$, $\left(\left(T I D_{i}, T I D_{j}, I D_{s}, R_{u}, t_{i}\right)\left(K_{u}\right)\right)$, $\left(\left(T I D_{i}, T I D_{j}, R_{i}, R_{j}, P_{i}, P_{j}, S K_{u s}, C_{s u}\right)\right)$, $\left(T I D_{i}, T I D_{j}, P_{i}, P_{j}, R_{i}, R_{j}, K_{i}, S K_{i j}\right)$. For each query $\left(\left(T I D_{u}, I D_{s}, R_{u}, X_{u}, V_{u}\right)\right)$, $\left(\left(T I D_{i}, T I D_{j}, I D_{s}, R_{u}, t_{i}\right)\right)$, $\left(\left(T I D_{i}, T I D_{j}, R_{i}, R_{j}, P_{i}, P_{j}, S K_{u s}\right)\right)$, $\left(\left(T I D_{i}, T I D_{j}, P_{i}, P_{j}, R_{i}, R_{j}\right)\right)$ issued by adversary A to the oracle, $C$ either returns a previous value else choose a number $S K_{i} \in Z_{p}^{*}$ such that there is no item

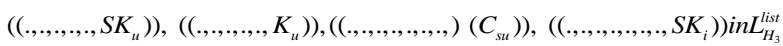
and returns $\left(\left(S K_{u}, K_{u}, C_{s u}, S K_{i}\right)\right)$ to A. Now $\mathrm{C}$ inserts the tuple $\left(\left(T I D_{u}, I D_{s}, R_{u}, X_{u}, V_{u}, S K_{u}\right)\right)$, $\left(\left(T I D_{i}, T I D_{j}, I D_{s}, R_{u}, t_{i},\right)\left(K_{u}\right)\right)$, $\left(\left(T I D_{i}, T I D_{j}, R_{i}, R_{j}, P_{i}, P_{j}, S K_{u s}, C_{s u}\right)\right)$, $\left(\left(T I D_{i}, T I D_{j}, P_{i}, P_{j}, R_{i}, R_{j}, K_{i}, S K_{i j}\right)\right)$ in the $\left(L_{H_{3}}^{l i s t}\right)$.

Registration: Initially, C holds an empty list $\left(L_{R}^{\text {list }}\right)$ consisting of tuples in the form $\left(\left(I D_{u}, r_{u}, R_{u}\right)\right)$, । $\left(\left(H_{2}\left(d_{s}\right), H_{2}\left(I D_{u}\right), T I D_{u}\right)\right)$. If the adversary A asks for Registration query with $\left(I D_{u}, T I D_{u}\right), \mathrm{C}$ searches the list $\left(L_{H_{1}}^{\text {list }}\right)$. If a tuple $\left(\left(I D_{u}, I D_{s}, R_{u},\right)\left(h_{u}\right)\right), \quad\left(\left(d_{s}, I D_{u}, T I D_{u}\right)\right)$ is found then $\mathrm{C}$ does nothing else $\mathrm{C}$ selects $\left(r_{u}, h_{u}, d_{s} \in Z_{p}^{*}\right)$ and compute $\left(r_{u}=U_{u}-h_{u} \cdot d_{s}\right)$, $\left(R_{u}=r_{u} \cdot P\right),\left(\left(H_{2}\left(d_{s}\right)=T I D_{u} \oplus H_{2}\left(I D_{u}\right)\right)\right.$ and answers as follows: 
1. If identity of user $I D_{u}=I D_{c}, \quad$ for $\left(I D_{u}=I D_{a}, I D_{b}\right), \mathrm{C}$ returns $\left(\left(I D_{u}, \perp, r_{c} \cdot P\right)\right)$ to A.

2. Else, the algorithm $\mathrm{C}$ returns $\left(\left(I D_{u}, r_{u}, R_{u}\right)\right)$ to A.

3. If $\mathrm{C}$ tries to act as $\mathrm{S}=\mathrm{C}$, then $\mathrm{C}$ returns $\left(\left(H_{2}\left(I D_{u}\right), \perp, d_{a}, T I D_{u}\right)\right)$ to $\mathrm{A}$.

4. Else, the returns $\left(\left(H_{2}\left(d_{s}\right), H_{2}\left(I D_{u}\right), T I D_{u}\right)\right)$ to user $\mathrm{A}$.

Finally, C inserts the tuple $\left(\left(d_{s}, I D_{u}, T I D_{u}\right)\right)$,

$\left(\left(I D_{u}, r_{u}, R_{u}\right)\right),\left(\left(H_{2}\left(d_{s}\right), H_{2}\left(I D_{u}\right), T I D_{u}\right)\right)$ into the list $\left(L_{H_{1}}^{\text {list }}\right),\left(L_{H_{2}}^{\text {list }}\right)$ and $\left(L_{R}^{\text {list }}\right)$.

Signature queries: Suppose A submits a signature query with sender's identity $\mathrm{ID}_{\mathrm{a}}$ and the receiver's identity $\mathrm{ID}_{\mathrm{b}}$, and then he searches the $L_{H_{1}}^{\text {list }}$ list then generates the signature as given below:

1. If $\left(T I D_{u} \neq I D_{A}\right)$ or $\mathrm{ID}_{\mathrm{b}}$, then $\mathrm{C}$ selects a random integers $x_{a}, x_{b} \in Z_{p}^{*}$ then compute the signature as $\quad\left(\sigma_{c}=\left(x_{a}+t_{i}\right) P_{b}\right)$ where $\left(P_{b} \in\{\right.$ Receiver $\left.\}\right)$ is the public key of user $\operatorname{ID}_{\mathrm{u}}$.

2. Otherwise, outputs abort the protocol.

Finally, $\mathrm{C}$ returns a signature $\left(\sigma_{c}\right)$ to A for the signer $T I D_{a}$ and the verifier $\left(I D_{b}\right)$.

Verify queries: When A makes this query to $\mathrm{C}$ for the verification of the signature $\left(\sigma_{c}\right)$ for the signer $\mathrm{TID}_{\mathrm{a}}$ and the verifier $\mathrm{TID}_{\mathrm{b}}, \mathrm{C}$ first checks whether $T I D_{a}, T I D_{b}=T I D_{i}, T I D_{j}$ holds.

1. If it holds, $\mathrm{C}$ terminates the session and reports abort $\perp$.

2. Else, recovers the secret key of receiver $x_{b}$ of $\operatorname{TID}_{b}$ and verifies the algorithm using the verification algorithm.

Send $\left(\pi_{a, b}^{s}, m\right)$ : Initially, $\mathrm{C}$ holds an empty list $\left(L_{S}^{\text {list }}\right)$ consisting of $\left(\pi_{a, b}^{s}, \operatorname{Trans}_{a, b}^{s}, N_{i}\right)$ where Trans $_{a, b}^{s}$ is the transcript of $\pi_{a, b}^{s}, \mathrm{C}$ answers the query as follows:
$1 \%$ OFMC

$1 \%$ Version of 2006/02/13

SUMMARY

SAFE

DETAILS

BOUNDED \NUMBER \OFISSESSIONS

PROTOCOL

C:ISPANltestsuitelresultsledited.if

GOAL

as\_specified

BACKEND

OFMC

COMMENTS

STATISTICS

parseTime: $0.00 \mathrm{~s}$

searchTime: 1.56

visitedNodes: 499 nodes

depth: 9 plies

Figure 1 Simulation result of OFMC

\section{SUMMARY}

SAFE

\section{DETAILS

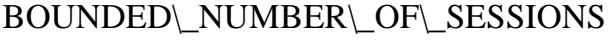 TYPED $\_M O D E L$}

PROTOCOL

C:ISPAN|testsuitelresultsledited.if

GOAL

As Specified

BACKEND

CL-AtSe

STATISTICS

Analysed :0 states

Reachable : 0 states

Translation: 0.08 seconds

Computation: 0.00 seconds

Figure 2 Simulation result of CL-AtSe

1. If $\left(\left(\pi_{a, b}^{s}=\pi_{a, b}^{T}\right)\right)$ and $m$ is the first message, $\mathrm{C}$ searches for the tuple in the form $\left(\left(t_{i}, \sigma_{i}, N_{i}\right)\right)$ into the $\left(L_{S}^{\text {list }}\right)$. Later, $\mathrm{C}$ inserts the tuple $\left(\pi_{a, b}^{T}, \operatorname{Trans}_{a, b}^{T}, N_{i}\right)$ to the list $\left(L_{S}^{\text {list }}\right)$.

2. Else, C looks for $\left(t_{i}, \sigma_{i}, N_{i}\right)$ into $\left(L_{S}^{\text {list }}\right)$, chooses a nonce $N_{r} \in Z_{p}^{*}$ and returns $\left(N_{r}, \sigma_{i}, T_{i}\right)$ as the 
Susmita Mandal et al.

answer. $\mathrm{C}$ updates the tuple indexed by $(\pi)_{a, b}^{s}$ in the list $\left(L_{S}^{\text {list }}\right)$.

Corrupt: This query is responded by $\mathrm{C}$ for searching a long lived key as follows:

1. If the identity of user $\left(T I D_{u}=T I D_{i}\right)$ or $\left(T I D_{j}\right),(C)$ aborts.

2. Else, the algorithm $\mathrm{C}$ searches for the list $\left(L_{R}^{\text {list }}\right)$

$$
\begin{array}{ll}
\text { for } & \operatorname{tuple}\left(\left(I D_{u}, r_{u}, R_{u}\right)\right), \\
\left(\left(H_{2}\left(d_{s}\right), H_{2}\left(I D_{u}\right), T I D_{u}\right)\right) \text { and } & \text { return } \\
\left(r_{u}, H_{2}\left(d_{s}\right)\right) \text { to A. } &
\end{array}
$$

$\operatorname{Reveal}\left(\pi_{a, b}^{s}, m\right)$ : Initially $\mathrm{C}$ holds an empty list $L_{R V L}^{\text {list }}$ containing the tuples in the form $\left(I D_{s}, I D_{r}, \pi_{a, b}^{s}, T_{a, b}^{s}, S K_{a, b}^{s}\right)$, where $\mathrm{ID}_{\mathrm{s}}$ in the identification of the sender in the session which $\pi_{a, b}^{s}$ and $\mathrm{ID}_{\mathrm{r}}$ represents the receiver identification. $\mathrm{C}$ answers the query as follows:

1.If $\left(\pi_{a, b}^{s}=\pi_{a, b}^{T}\right)$, then $\mathrm{C}$ aborts the session.

2.If $\left(I D_{u} \neq I D_{a}\right)$ or $\mathrm{ID}_{\mathrm{b}}$ then $\mathrm{C}$ searches in the lists $L_{S}^{\text {list }}, L_{R}^{\text {list }}$ and $\quad L_{H_{1}}^{\text {list }}$ for corresponding tuples $\left(\left(\pi_{a, b}^{s}, \operatorname{Trans}_{a, b}^{s}, N_{i}\right)\right),\left(\left(I D_{u}, r_{u}, R_{u}\right)\right)$, $\left(H_{2}\left(d_{s}\right), H_{2}\left(I D_{u}\right),\left(T I D_{u}\right)\right.$ $\left(\left(I D_{u}, I D_{s}, R_{u}, h_{u}\right)\right),\left(\left(V_{u}, U_{u}, Z_{u}\right)\right)$, $\left(\left(x_{u}, I D_{u}, X_{u}\right)\right)$, and $\left(K_{i j}, N_{i}, N_{j}, R_{i}, R_{j}, \sigma_{u}, K_{i}\right)_{\text {receptively. }}$ Then C computes $K_{a, b}^{s}=\left(x_{i} \cdot P_{u b}\right)$.

1. $\mathrm{C}$ makes a $\mathrm{H}_{3}$ query. If $\left(\pi_{a, b}^{s}\right)$ is the sender oracle A query as $\left(T I D_{i}, T I D_{j}, P_{i}, P_{j}, R_{i}, R_{j}, K_{i}\right)$.
2. Else, C chooses $S K_{a, b}^{s} \in Z_{p}^{*}$.

Test $\left(\pi_{a, b}^{T}\right)$ : This query is allowed once the adversary asks any of the above mentioned queries for which the oracle $\left(\pi_{a, b}^{T}\right)$ must be fresh. If it does not choose any oracles then $\mathrm{C}$ aborts the session. Else, it randomly responds with a value from the set $\left(Z_{p}^{*}\right)$.

\subsection{AVISPA}

AVISPAs a push-button tool for the automated validation of the Internet security-sensitive protocols and applications [19]. It is considered as a widely accepted simulation tool for formal security verification, which measured whether the security protocol is $S A F E$ or UNSAFE. It uses a special language called High-Level Protocol Specification Language and integrates the different back-ends that implement a variety of state-of-the-art automatic analysis techniques [20].

The architecture of AVISPA is demonstrated in Figure 3 This language is based on roles where the AVISPA tool mechanically translates the HLPSL into a lower level specification using a HLPSL2IF translator. Afterward, it generates an intermediate format (IF). The present version integrates four backends namely, on-the-fly model-checker (OFMC), CLbased attack searcher (CL-AtSe), SAT-based modelchecker (SATMC), and tree-automata-based protocol analyzer (TA4SP).

The OFMC is responsible for symbolic techniques for exploring the state place in a demand driven way. CL-AtSe provides a translation from any security protocol wrote into an intermediate format IF into a set of constraints mainly used to find whether there are attacks on protocols. SAT generates a propositional form then input into a SAT solver and any model found is translated back into an attack.

Finally, TA4SP is responsible for approximating the intruder knowledge using regular tree languages. 


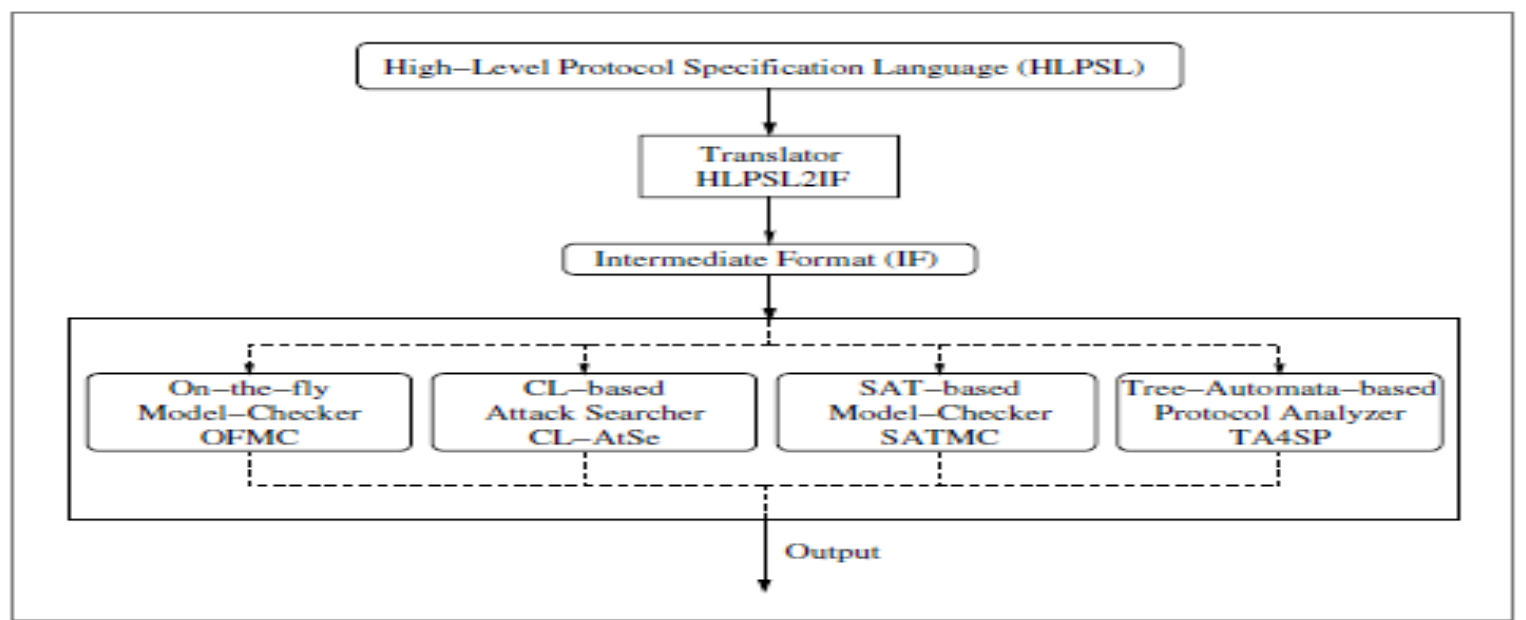

Figure 3 Architecture of AVISPA

Table 1 Security comparisons of the protocol with others

\begin{tabular}{|c|c|c|c|c|c|}
\hline Attribute & $\begin{array}{l}\text { Ya } \\
\text { ng } \\
\text { Et } \\
\text { Al. }\end{array}$ & $\begin{array}{l}\text { Pu } \\
\text { Et } \\
\text { Al. }\end{array}$ & $\begin{array}{l}\text { Tan } \\
\text { Et } \\
\text { Al. }\end{array}$ & $\begin{array}{l}\text { Islam } \\
\text { Et Al. }\end{array}$ & $\begin{array}{l}\text { Our } \\
\text { Schem } \\
\text { e }\end{array}$ \\
\hline Anonymity & No & No & No & No & Yes \\
\hline $\begin{array}{l}\text { Impersonation } \\
\text { attack }\end{array}$ & No & No & No & Yes & Yes \\
\hline $\begin{array}{l}\text { Non- } \\
\text { repudiation }\end{array}$ & No & No & No & No & Yes \\
\hline $\begin{array}{l}\text { Parallel } \\
\text { session attack }\end{array}$ & No & Yes & Yes & Yes & Yes \\
\hline $\begin{array}{l}\text { Man-in-the- } \\
\text { middle-attack }\end{array}$ & No & No & No & Yes & Yes \\
\hline $\begin{array}{l}\text { Perfect } \\
\text { forward } \\
\text { secrecy }\end{array}$ & Yes & Yes & Yes & Yes & Yes \\
\hline $\begin{array}{l}\text { Known-key } \\
\text { security }\end{array}$ & Yes & Yes & Yes & Yes & Yes \\
\hline $\begin{array}{l}\text { Server- } \\
\text { impersonation } \\
\text { attack }\end{array}$ & No & No & No & No & Yes \\
\hline
\end{tabular}

4.2.1Analysis and specification of result

We have implemented our scheme using the HLPSL language where, we assign three primary roles namely, alice, server, and bob represented as A, S and $\mathrm{B}$ respectively. The results ensure that our proposed scheme is safe under OFMC and CL-AtSe back-ends. It is tested using SPAN for AVISPA in Figure 1 and Figure 2. The results ensure that our scheme can withstand popular active attacks, such as the masquerading, replay, and man-in-the-middle attacks, and passive attacks.

\subsection{BAN Logic}

BAN is logic of authentication proposed by BurrowsAbadi-Needham [21, 22].

The goal of BAN logic is to provide trust among communicating parties. It is used to analyse the security of authentication and key distribution protocols. In this section, we first briefly describe the notations used in the $\backslash$ (BAN $\backslash$ ) logic, and after that, we provide the authentication proof.

The notations of the BAN logic are as follows:

- $\{\mathrm{P}, \mathrm{Q}\}$ : are the participating entities.

- $\{\mathrm{X}\}$ : message sends in channel.

- $\{\mathrm{K}\}$ : the secret key.

- $\{X\}_{K}$ : message is encrypted with the secret key.

- $P \mid \equiv Q: \mathrm{P}$ believes in $\mathrm{Q}$.

- $P \triangleleft X: \mathrm{P}$ received message $\mathrm{X}$.

- $P \mid \sim X: P$ once said $X$.

- $Q \Rightarrow X: \mathrm{Q}$ has jurisdiction on $\mathrm{X}$.

- $\#(X): \mathrm{X}$ is fresh.

$$
K
$$

- $P \stackrel{K}{\leftrightarrow} Q: \mathrm{K}$ is a shared key between $\mathrm{P}$ and $\mathrm{Q}$.

Some rules used in BAN logic as follows:

Message-meaning rule: $\frac{P \mid P \stackrel{K}{\leftrightarrow} Q, P \triangleleft\{X\}_{K}}{P|\equiv Q| \sim X}$

Nonce-verification rule: $\frac{P|\equiv \#(X), P| \equiv Q \mid \sim X}{P|\equiv Q| \equiv X}$

Jurisdiction rule: $\frac{P|\equiv Q \Rightarrow X, P| \equiv Q \mid \equiv X}{P \mid \equiv X}$

Belief-joint rule: $\frac{P \mid \equiv Q \sim(X, Y)}{P \mid \equiv Q \sim X}$ 
Susmita Mandal et al.

Freshness-joint rule: $\frac{P \mid \equiv \#(X)}{P \equiv \#(X, Y)} \quad B \mid \equiv B \stackrel{\mathrm{K}_{\mathrm{sb}}}{\leftrightarrow} S$

Additional rule: $\frac{\#(K), P \triangleleft\{X\}_{K}, P \mid \equiv P \stackrel{K}{\leftrightarrow} Q}{\stackrel{K}{\leftrightarrow} Q} \quad B \mid \equiv A \stackrel{\mathrm{K}_{\mathrm{ab}}}{\leftrightarrow} A$

$P|\equiv Q| \sim X, P|\equiv Q| \equiv P \stackrel{K}{\leftrightarrow} Q \quad B \mid \Rightarrow S K_{b s}$

Deduction of the proposed protocol

$$
B|\equiv S| \stackrel{\mathrm{C}_{\mathrm{sb}}}{\leftrightarrow} S
$$

\section{Idealization}

$M S 2: A \rightarrow S, I D_{s}, T I D_{b}, T_{1},\left\{V_{a}, M_{a}, F_{a}, S K_{a s}\right\}_{K_{a}}$
$M S 4: B \rightarrow S, I D_{s}, T I D_{a}, T_{2},\left\{V_{b}, M_{b}, F_{b}, S K_{b s}\right\}_{K_{b}}$
$M S 5: S \rightarrow A, I D_{s}, T I D_{b}, P u b,\left\{R_{b}, C_{s a}\right\}_{K_{s a}}$
$M S 6: S \rightarrow B, I D_{s}, T I D_{a}, P u a,\left\{R_{a}, C_{s b}\right\}_{K_{s b}}$
$M S 7: S \rightarrow A, T I D_{a}, T I D_{b},\left\{T_{3}, \sigma_{a}, N_{b}\right\}_{K_{a b}}$

Note: MS1, MS3 does not contribute to the logic therefore it is omitted from the proof.

\section{Initial state assumption}

The initial state assumption of $\mathrm{S}$ are:

$$
\begin{aligned}
& S \mid \equiv S \stackrel{\mathrm{K}_{\mathrm{a}}}{\leftrightarrow} A \\
& S \mid \equiv S \stackrel{\mathrm{K}_{\mathrm{b}}}{\leftrightarrow} B \\
& S \mid \equiv S \stackrel{\mathrm{K}_{\mathrm{sa}}}{\leftrightarrow} A \\
& S \mid \equiv S \stackrel{\mathrm{K}_{\mathrm{sb}}}{\leftrightarrow} B \\
& S|\equiv A| \Rightarrow S \stackrel{\mathrm{SK}_{\mathrm{as}}}{\leftrightarrow} A \\
& S|\equiv B| \Rightarrow S \stackrel{\mathrm{SK}_{\mathrm{bs}}}{\leftrightarrow} B \\
& S \mid \Rightarrow C_{s a} \\
& S \mid \Rightarrow C_{s b}
\end{aligned}
$$

The initial state assumption of A are:

$$
\begin{aligned}
& A \equiv A \stackrel{\mathrm{K}_{\mathrm{a}}}{\leftrightarrow} S \\
& A \equiv A \stackrel{\mathrm{K}_{\mathrm{sa}}}{\leftrightarrow} S \\
& A \equiv A \stackrel{\mathrm{K}_{\mathrm{ab}}}{\leftrightarrow} B \\
& A \mid S K_{a s} \\
& A \mid \equiv S \mapsto A \stackrel{\mathrm{C}_{\mathrm{sa}}}{\leftrightarrow} S
\end{aligned}
$$

\section{Annotation}

$$
\begin{aligned}
& S \triangleleft\left\{V_{a}, M_{a}, F_{a}, A \stackrel{\mathrm{SK}_{\mathrm{as}}}{\leftrightarrow} S, \#\left(A \stackrel{\mathrm{SK}_{\mathrm{as}}}{\leftrightarrow} S\right)\right\}_{K_{a}} \\
& S \triangleleft\left\{V_{b}, M_{b}, F_{b}, B \stackrel{\mathrm{SK}_{\mathrm{bs}}}{\leftrightarrow} S, \#\left(B \stackrel{\mathrm{SK}_{\mathrm{bs}}}{\leftrightarrow} S\right)\right\}_{K_{b}} \\
& A \triangleleft\left\{R_{b}, A \stackrel{\mathrm{C}_{\mathrm{sa}}}{\leftrightarrow} S, \#\left(A \stackrel{\mathrm{C}_{\mathrm{sa}}}{\leftrightarrow} S\right)\right\}_{K_{s a}} \\
& B \triangleleft\left\{R_{a}, B \stackrel{\mathrm{C}_{\mathrm{sb}}}{\leftrightarrow} S, \#\left(B \stackrel{\mathrm{C}_{\mathrm{sb}}}{\leftrightarrow} S\right)\right\}_{K_{s b}} \\
& B \triangleleft\left\{T_{3}, \sigma_{a}, N_{b}, A \stackrel{\mathrm{K}_{\mathrm{ab}}}{\leftrightarrow} B\right\}
\end{aligned}
$$

\section{Derivation process}

According to MS2

$$
\begin{aligned}
& \frac{S \mid S \stackrel{\mathrm{K}_{\mathrm{a}}}{\leftrightarrow} A, S \triangleleft\left\{V_{a}, M_{a}, F_{a}, S K_{a s}\right\}_{K_{a}}}{S \mid \equiv A \sim\left\{V_{a}, M_{a}, F_{a}, S K_{a s}\right\}} \\
& \frac{S|\equiv A| \sim\left\{V_{a}, M_{a}, F_{a}, S K_{a s}\right\}}{S \mid \equiv A \sim\left\{S K_{a s}\right\}} \\
& \frac{S|\equiv A| \sim\left\{S K_{a s}\right\}, S|\equiv A| S K_{a s}}{S \mid S K_{a s}}
\end{aligned}
$$

According to MS4

$$
\begin{aligned}
& \frac{S \mid S \stackrel{\mathrm{K}_{\mathrm{b}}}{\leftrightarrow} B, S \triangleleft\left\{V_{b}, M_{b}, F_{b}, S K_{b s}\right\}_{K_{b}}}{S \mid \equiv B \sim\left\{V_{b}, M_{b}, F_{b}, S K_{b s}\right\}} \\
& \frac{S|\equiv B| \sim\left\{V_{b}, M_{b}, F_{b}, S K_{b s}\right\}}{S \mid \equiv B \sim\left\{S K_{b s}\right\}} \\
& \frac{S|\equiv B| \sim\left\{S K_{b s}\right\}, S|\equiv B| S K_{b s}}{S \mid S K_{b s}}
\end{aligned}
$$

According to MS5

$$
\begin{aligned}
& \frac{A \equiv A \stackrel{\mathrm{K}_{\mathrm{sa}}}{\leftrightarrow} S, A \triangleleft\left\{R_{a}, C_{s a}\right\}_{K_{s a}}}{A \mid \equiv S \sim\left\{R_{b}, C_{s a}\right\}} \\
& \frac{A \equiv S \mid \sim\left\{R_{b}, C_{s a}\right\}}{A \equiv S \sim\left\{C_{s a}\right\}}
\end{aligned}
$$

The initial state assumption of $\mathrm{B}$ are:

$$
B \mid \equiv B \stackrel{\mathrm{K}_{\mathrm{b}}}{\leftrightarrow} S
$$




$$
\frac{A|\equiv S| \sim\left\{C_{s a}\right\}, A|\equiv S| \Rightarrow C_{s a}}{A \mid \equiv C_{s a}}
$$

According to MS6

$$
\begin{aligned}
& \frac{B \mid \equiv B \stackrel{\mathrm{K}_{\mathrm{sb}}}{\leftrightarrow} S, B \triangleleft\left\{R_{a}, C_{s b}\right\}_{K_{s b}}}{B \mid \equiv S \sim\left\{R_{a}, C_{s b}\right\}} \\
& \frac{B|\equiv S| \sim\left\{R_{a}, C_{s b}\right\}}{B \mid \equiv S \sim\left\{C_{s b}\right\}} \\
& \frac{B|\equiv S| \sim\left\{C_{s b}\right\}, B|\equiv S| \Rightarrow C_{s b}}{B \mid \equiv C_{s b}}
\end{aligned}
$$

According to MS7

$$
\begin{aligned}
& \frac{B \mid \equiv B \leftrightarrow A, B \triangleleft\left\{T_{3}, \sigma_{a}, N_{b}\right\}_{K_{a b}}}{B \mid \equiv A \sim\left\{T_{3}, \sigma_{a}, N_{b}\right\}} \\
& \frac{B \mid \equiv \#\left(N_{b}\right)}{B \mid \equiv \#\left\{N_{b}, T_{3}, \sigma_{a}\right\}} \\
& \frac{B|\equiv A| \sim\left\{N_{b}, T_{3}, \sigma_{a}\right\}, B \mid \equiv \#\left\{N_{b}, T_{3}, \sigma_{a}\right\}}{B|\equiv A| \equiv\left\{N_{b}, T_{3}, \sigma_{a}\right\}} \\
& \frac{\# K_{a b}, B\left|\triangleleft\left\{N_{b}, T_{3}, \sigma_{a}\right\}_{K_{a b}}, B\right| \equiv A \stackrel{\mathrm{K}_{\mathrm{ab}}}{\leftrightarrow} B}{\left.\stackrel{\mathrm{K}_{\mathrm{ab}}}{\leftrightarrow}\right) B} \\
& B|\equiv A| \sim\left(N_{b}\right), B|\equiv A| \equiv A \stackrel{\leftrightarrow}{\leftrightarrow}
\end{aligned}
$$

\section{Performance evaluation}

In this section, we compare the proposed scheme with some existing systems already discussed in the literature with respect to computation cost efficiency.

Table 2 Conversion of various operations

\begin{tabular}{ll}
\hline Notations & Definition and conversion \\
\hline$T_{M L}$ & $\begin{array}{l}\text { Time complexity for executing the modular } \\
\text { multiplication }\end{array}$ \\
$T_{P M}$ & $\begin{array}{l}\text { Time complexity for executing the elliptic } \\
\text { curve scalar point multiplication } \\
\end{array}$ \\
& $T_{P M} \approx 29 T_{M L}$ \\
& Time complexity for executing the billinear \\
$T_{B P}$ & pairing operation, $T_{B P} \approx 87 T_{M L}$ \\
& \& Time complexity for executing the \\
$T_{I N}$ & modular $\quad$ inversion \\
& $T_{I N} \approx 11.6 T_{M L}$ \\
$T_{H}$ & Time complexity for executing the hash \\
& function is negligible
\end{tabular}

In Table 1, we represent the security comparison of existing schemes with ours whereas, Table 2. enlist the conversion notations of various operation units with respect to modular multiplication. The computation cost of a symmetric en/decryption algorithm almost takes same computation cost as a hash function therefore it can be overlooked with the ECC point multiplication. The comparison is depicted in Table 3.

Table 3 Comparison of communication cost in each protocol

\begin{tabular}{lllll}
\hline Protocol & $\begin{array}{l}\text { Us } \\
\text { er } \\
\mathbf{A}\end{array}$ & $\begin{array}{l}\text { User } \\
\mathbf{B}\end{array}$ & Server $\mathbf{S}$ & Total cost \\
\hline Yang et al. & & $5 T_{P M}$ & $2 T_{P M}$ & $12 T_{P M} \approx 348 \$ T_{M L}$ \\
& $5 T_{P M} 5 T_{P M}$ & $2 T_{P M}$ & $12 T_{P M} \approx 348 \$ T_{M L}$ \\
Pu et al. & & & \\
Tan et al. & $3 T_{P M} 3 T_{P M}$ & $T_{P M}+4 T_{B P}$ & $8 T_{P M}+4 T_{B P}$ \\
& & & $\approx 580 T_{M L}$ \\
$\begin{array}{l}\text { Islam et } \\
\text { al. }\end{array}$ & $4 T_{M L} 4 T_{M L}$ & $2 T_{M L}$ & $10 T_{P M} \approx 290 T_{M L}$ \\
\multicolumn{1}{c}{ Our } & $3 T_{P M} 3 T_{P M}$ & $2 T_{P M}$ & $8 T_{P M} \approx 232 T_{M L}$ \\
Scheme & & & \\
\hline
\end{tabular}

\section{Conclusion}

This paper proposes an ID-based authenticated threeparty key exchange protocol based on elliptic curve cryptography, where the security of the session lies on ECDHP assumption in the random oracle. The scheme achieves anonymity and non-repudiation with efficiency. Furthermore, the overall computation cost is lesser than existing schemes; also it is validated in AVISPA toolkit and formally verified using BAN logic. The results confirmed that the proposed scheme is secure under OFMC and CL-AtSe backends.

\section{Acknowledgment}

None.

Conflicts of interest

The authors have no conflicts of interest to declare.

\section{References}

[1] Bellovin SM, Merritt M. Encrypted key exchange: Password-based protocols secure against dictionary attacks. In proceedings of IEEE computer society symposium on research in security and privacy 1992 (pp. 72-84). IEEE.

[2] Bellare M, Rogaway P. Entity authentication and key distribution. In annual international cryptology conference 1993 (pp. 232-49). Springer Berlin Heidelberg. 
[3] Bellare M, Pointcheval D, Rogaway P. Authenticated key exchange secure against dictionary attacks. In international conference on the theory and applications of cryptographic techniques 2000 (pp. 139-55). Springer Berlin Heidelberg.

[4] Choie YJ, Jeong E, Lee E. Efficient identity-based authenticated key agreement protocol from pairings. Applied Mathematics and Computation. 2005; 162(1):179-88.

[5] Chen TH, Lee WB, Chen HB. A round-and computation-efficient three-party authenticated key exchange protocol. Journal of Systems and Software. 2008; 81(9):1581-90.

[6] Yang JH, Chang CC. An efficient three-party authenticated key exchange protocol using elliptic curve cryptography for mobile-commerce environments. Journal of Systems and Software. 2009; 82(9):1497-502.

[7] Schnorr CP. Efficient signature generation by smart cards. Journal of Cryptology. 1991; 4(3):161-74.

[8] Pu Q, Zhao X, Ding J. Cryptanalysis of a three-party authenticated key exchange protocol using elliptic curve cryptography. In international conference on research challenges in computer science 2009 (pp. 710). IEEE.

[9] Tan Z. An improvement on a three-party authentication key exchange protocol using elliptic curve cryptography. Journal of Convergence Information Technology. 2010; 5(4):120-9.

[10] Nose P. Security weaknesses of authenticated key agreement protocols. Information Processing Letters. 2011; 111(14):687-96.

[11] Lin CL, Sun HM, Hwang T. Three-party encrypted key exchange: attacks and a solution. ACM SIGOPS Operating Systems Review. 2000; 34(4):12-20.

[12] Chang CC, Chang YF. A novel three-party encrypted key exchange protocol. Computer Standards \& Interfaces. 2004; 26(5):471-6.
[13] Lu R, Cao Z. Simple three-party key exchange protocol. Computers \& Security. 2007; 26(1):94-7.

[14] Farash MS, Attari MA. A secure and efficient identitybased authenticated key exchange protocol for mobile client-server networks. The Journal of Supercomputing. 2014; 69(1):395-411.

[15] Shamir A. Identity-based cryptosystems and signature schemes. In workshop on the theory and application of cryptographic techniques 1984 (pp. 47-53). Springer Berlin Heidelberg.

[16] Miller VS. Use of elliptic curves in cryptography. In conference on the theory and application of cryptographic techniques 1985 (pp. 417-26). Springer Berlin Heidelberg.

[17] Koblitz N. Elliptic curve cryptosystems. Mathematics of Computation. 1987; 48(177):203-9.

[18] Bellare M, Rogaway P. Random oracles are practical: A paradigm for designing efficient protocols. In proceedings of the ACM conference on computer and communications security 1993 (pp. 62-73). ACM.

[19] AVISPA Web tool: automated validation of internet security protocols and applications. www.avispaproject.org/web-interface/. Accessed 16 June 2016.

[20] HLPSL tutorial, 2006. http://www.avispaproject.org/package/tutorial.pdf. Accessed 16 June 2016.

[21] Burrows M, Abadi M, Needham RM. A logic of authentication. In proceedings of the royal society of London a: mathematical, physical and engineering sciences 1989 (pp. 233-71). The Royal Society.

[22] Wen J, Zhang M, Li X. The study on the application of BAN logic in formal analysis of authentication protocols. In Proceedings of the 7th international conference on electronic commerce 2005 (pp. 744-7). ACM.

This paper is selected from proceedings of National Workshop on Cryptology-NWC 2016 organized at JNN College of Engineering Shimoga, Karnataka, India during 11-13, August 2016. 